\title{
Nouvelles aventures franco-ontariennes : quelle relève pour la littérature pour la jeunesse en Ontario français?
}

\author{
Camylle Gauthier-Trépanier \\ Université d'Ottawa
}

La littérature pour la jeunesse franco-ontarienne naît dans les années 1970, avec la création des premières maisons d'édition de langue française en Ontario. On note toutefois la publication de contes et de récits folkloriques destinés aux enfants avant cette période. C'est le cas des contes d'Emma Lacerte publiés par l'imprimerie Beauregard ${ }^{1}$ et Beauchemin éditeur (Lepage, 2000, p. 644) au cours de la première moitié du XXe siècle et de ceux de

\footnotetext{
${ }^{1}$ L'imprimerie Beauregard d'Ottawa consacre aussi une partie de ses activités à l'édition (Centre de recherche en civilisation canadienne française, 2003).
} 
CAMYLLE GAUTHIER-TRÉPANIER, « Nouvelles aventures franco-ontariennes : quelle relève pour la littérature pour la jeunesse en Ontario français? »

Marie-Rose Turcot, publiés chez Beauchemin éditeur et Fidès (Lepage, 2000, p. 742). Il s'agit de textes dont la vocation est principalement éducative. Notons également le travail du père Germain Lemieux qui a compilé un grand nombre de contes et de chansons dans les volumes de Les vieux m'ont conté, publiés par le Centre franco-ontarien de folklore, dont le premier tome paraît en 1973. Ainsi, bien que les années 1970 constituent la date de « naissance » officielle, tant de la littérature qui s'affirme comme franco-ontarienne que de son pendant pour la jeunesse, ce n'est qu'à partir des années 1980 que l'on distingue véritablement une production éditoriale dont l'objectif est de rejoindre un jeune public et de lui plaire en évoquant des enjeux et un univers qui lui ressemble. À la différence de l'Ouest canadien et de l'Acadie où l'album s'impose comme la forme la plus commune de littérature pour la jeunesse, c'est le roman qui domine la production en Ontario français avec des auteurs tels que Doric Germain, Paul Prud'homme, Ginette Proulx-Weaver et Jean-Louis Grosmaire (Lepage, 2000, p. 431-433). On peut également citer les romans d'Hélène Brodeur (La quête d'Alexandre, 1981; Entre l'aube et le jour, 1983; Les routes incertaines, 1886) qui n'ont pas, originellement, été publiés pour les jeunes, mais qui ont rapidement été récupérés par l'institution scolaire puisqu'ils permettaient d'enseigner l'histoire de l'Ontario.

Au début des années 2000, on compte quatre maisons d'éditions franco-ontariennes actives dans le secteur de la littérature pour la jeunesse, soit les éditions du Vermillon, Prise de parole, Pierre de lune et l'Interligne. En date de 2005, seules « les éditions du Vermillon et les éditions l'Interligne, publient régulièrement des livres pour la jeunesse ». De ces deux maisons, Vermillon «se taill[e] la part du lion en publiant l'essentiel de la production de quelques nouveaux auteurs, mais surtout d'écrivains qui lui sont fidèles depuis leurs débuts » (Lepage, 2005, p. 71) en 1981. Dans son article «Où en est la littérature franco-ontarienne 
pour la jeunesse? », Françoise Lepage recense un total de 32 ouvrages destinés à la jeunesse parus en Ontario entre 2002 et 2005. De 2006 à 2009, on ne note que 22 titres. Entre 2010 et 2013, inclusivement, ce nombre grimpe à 41, ce qui représente une augmentation de 10 titres, soit 28\% par rapport à la production recensée par Lepage. Par rapport aux années précédente, cela représente presque le double de la production, soit 19 titres ou une augmentation de $86 \%$. Cette augmentation s'explique notamment par l'arrivée d'un nouveau membre au sein du milieu de l'édition franco-ontarienne : les éditions David, fondées par Yvon Mallette en 1993. Les années 2000 marquent ainsi pour l'Ontario français le début d'une effervescence en matière de littérature pour la jeunesse. Les éditions du Vermillon continuent de publier avec régularité ${ }^{2}$. Aux éditions l'Interligne, la chercheuse et critique en littérature pour la jeunesse Françoise Lepage prend, en 2004, la tête de la collection jeunesse «Cavales » où elle va elle-même publier plusieurs ouvrages, dont Poupeska (2006) qui s'est mérité le prix du Gouverneur général. Quant à elles, les éditions David, mettent sur pied deux nouvelles collections : «14/18 » et «Voix didactiques ». La première regroupe des romans destinés aux adolescents et la seconde, chapeautée par Françoise Lepage, s'intéresse aux auteurs pour la jeunesse et à l'enseignement de ces œuvres. On y retrouve notamment des ouvrages consacrés à Doric Germain, Daniel Marchildon, et Hélène Brodeur, mais aussi à des auteurs québécois. Plus récemment, l'ajout de catégories jeunesse à divers prix littéraires francoontariens et franco-canadiens comme les prix Le Droit (1998), Trillium (2006), Champlain (2016) et Émergence-AAOF (2018) témoigne aussi de l'intérêt croissant qui se développe pour la littérature jeunesse franco-ontarienne.

\footnotetext{
${ }^{2}$ Toutefois leurs activités se sont réduites depuis 2013.
} 
CAMYLLE GAUTHIER-TRÉPANIER, « Nouvelles aventures franco-ontariennes : quelle relève pour la littérature pour la jeunesse en Ontario français? »

Ainsi, la création de nouvelles collections au tournant des années 2000 et l'augmentation du nombre de titres parus depuis 2010 témoignent à la fois de la vitalité de la littérature pour la jeunesse en Ontario et de la présence d'une relève dans ce secteur précis de l'industrie littéraire. Cela dit, le peu d'intérêt porté par les chercheurs à la littérature franco-ontarienne pour la jeunesse demeure problématique. Face à des espaces critiques peu nombreux, l'institution universitaire a également un rôle à jouer dans la diffusion et le rayonnement des œuvres franco-ontariennes pour la jeunesse. Nous tenterons donc d'amorcer la réflexion sur la relève dans la littérature franco-ontarienne pour la jeunesse en nous limitant au genre du roman d'aventure pour la jeunesse. En comparant des romans d'aventures récents au corpus utilisé par Françoise Lepage dans son Histoire de la littérature pour la jeunesse (2000), nous espérons cerner un certain nombre de caractéristiques propres à la relève dans le roman d'aventure franco-ontarien pour la jeunesse. Nous nous pencherons d'abord sur les composantes du roman d'aventure franco-ontarien tel que le concevait Françoise Lepage au moyen des romans de Doric Germain et de Jean-Louis Grosmaire, qu'elle inclut également dans le corpus franco-ontarien. Nous chercherons ensuite à tracer un portrait formel et thématique de deux romans d'aventure de la collection «14/18 » des éditions David parus après 2010 soit, 24 heures de liberté (2013) et Otages de la nature $(2018)^{3}$ dans le but de nous interroger sur le rapport qu'entretiennent ces œuvres récentes avec celles qui les ont précédées. Cela nous permettra par la suite de cerner les particularités qui définissent les textes de la relève, du moins en ce qui a trait au roman d'aventure.

\footnotetext{
${ }^{3}$ Nous avons choisi, pour des raisons de faisabilité, de nous restreindre au roman d'aventure et d'écarter ceux qui empruntaient à d'autres genres bien connus comme le roman historique (John et le règlement 17) ou la science-fiction (Ipod et minijupe au $18^{e}$ siècle, Projet Ithuriel) un critère qui n'a permis de retenir que des auteurs masculins.
} 


\section{Quelle relève pour le roman franco-ontarien pour la jeunesse?}

Dans son article « Où en est la littérature franco-ontarienne pour la jeunesse? », paru en 2005, Françoise Lepage évoque « une relève qui est loin d'être assurée » (p. 71) pour expliquer la précarité de la littérature pour la jeunesse franco-ontarienne. Plus qu'une continuité, c'est avant tout une assurance de viabilité que désire la chercheuse pour cette littérature qu'elle juge encore trop fragile et sous représentée. Le processus de consolidation de cette relève, qui prend le relais d'auteurs établis comme Doric Germain et Jean-Louis Grosmaire, semble,

pour Françoise Lepage, faire appel à des éléments qualitatifs. Éléments qui, pour Lucie Hotte et François Ouellet, semblent se raréfier dans les années 2000 (Hotte et Ouellet, 2016, p. 7). Interrogées à ce sujet par Françoise Lepage, les maisons d'édition Vermillon et l'Interligne soulignent effectivement la rareté des bons manuscrits et l'immense travail de correction auquel doivent s'atteler les éditeurs (2005, p. 71).

Les qualités de plume auxquelles font référence Lucie Hotte et François Ouellet relèvent d'éléments stylistiques appartenant au domaine de la forme. Dans le cas de la littérature pour la jeunesse, qui s'adresse à un lecteur en formation, plusieurs auteurs et éditeurs vont adapter les textes à leur lectorat en employant un vocabulaire ou des structures plus simples, déterminées par l'âge du lecteur. Le texte pour la jeunesse n'en est pas moins littéraire pour autant. Cependant, les qualités littéraires que l'on prête aux textes s'adressant à des lecteurs plus aguerris ne seront pas les mêmes que celles que l'on pourra retrouver dans les ouvrages destinés à la jeunesse. Dans ces derniers, on s'intéressera, par exemple, à la vraisemblance du langage employé par de jeunes personnages ou encore au développement de personnages auxquels le lecteur pourra s'identifier. Le style est donc, ici, un critère ambigu pour cerner les auteurs de la relève en littérature pour la jeunesse, il ne reste donc plus que 
CAMYLLE GAUTHIER-TRÉPANIER, « Nouvelles aventures franco-ontariennes : quelle relève pour la littérature pour la jeunesse en Ontario français? »

l'originalité des textes à considérer. Dans Les littératures de l'exiguïté François Paré explique effectivement que pour attirer l'attention de la critique, les œuvres littéraires devraient se distinguer par leur originalité. Toutefois,

[d]ans les petites ${ }^{4}$ cultures - en Ontario français, par exemple - il se publie bon an mal an bien des livres sans originalité aucune. On pourrait en dire autant des grandes institutions littéraires comme la France ou les États-Unis, mais l'abondance générale du discours littéraire, dans ces sociétés, masque plus facilement les cicatrices et les égratignures. Et les mécanismes institutionnels eux-mêmes, comme les recensements de livres, passent la banalité plus volontiers sous silence. Il y a dans ces mécanismes une extraordinaire efficacité d'occultation. Dans les petites cultures, au contraire, le seul fait de publier une œuvre semble mériter que la rumeur publique y fasse écho; on y devient écrivain reconnu très rapidement. Mais ce qu'on apprend vite, c'est que la notoriété en question est on ne peut plus évanescente[.] (Paré, 2001 [1992], p. 85)

Le fonctionnement de l'industrie littéraire dans les communautés francophones minoritaires biaiserait donc les mouvements naturels de la littérature empêchant de ce fait même l'émergence de nouvelles tendances qui se noieraient au milieu d'œuvres banales. Toutefois, l'originalité, tout comme la banalité et les « qualités de plume », sont des concepts subjectifs qu'il est difficile de baliser. Retenons donc que les chercheurs et critiques sont satisfaits ou intéressés par des œuvres existantes (ou plutôt par le style et l'originalité d'œuvres existantes) et qu'ils ont des attentes précises en matière de qualité. Les œuvres de la relève se mesureraient donc constamment à un horizon d'attente développé notamment au contact d'œuvres jugées importantes au sein d'une production littéraire particulière. L'appartenance à une forme de relève découlerait donc à la fois d'une capacité à construire sur la production précédente, contentant ainsi son public, mais également à la surpasser de quelque façon que ce soit. C'est-à-dire que l'écrivain.e de la relève devrait surpasser, ou en montrer le potentiel, ceux et celles qui l'ont précédé, tout en le dépassant.

\footnotetext{
${ }^{4}$ C'est au sens de minoritaire que François Paré emploie l'adjectif petite.
} 


\section{Le roman d'aventure et l'Ontario français : le début d'une tradition?}

Le roman d'aventure reflète de façon particulièrement pertinente l'ambivalence de la littérature pour la jeunesse franco-ontarienne qui est à la fois vivante, particulièrement depuis $2010^{5}$, mais peu étudiée dans le monde universitaire puisqu'il s'agit d'un genre fréquemment associé à la littérature dite populaire. Les définitions du roman d'aventure sont multiples, il est tout de même possible d'identifier un certain nombre d'éléments commun à l'ensemble de cette production littéraire, soit

une place centrale accordée à l'action et, de préférence, à l'action violente, une certaine dynamique du récit retranscrivant dans l'écriture cette relation à l'action, l'importance du dépaysement qui, sans être toujours central, paraît toujours jouer un rôle dans les œuvres (Letourneux, 2010, p. 13).

En Ontario français, il s'agit d'un genre présent pour les jeunes lecteurs depuis les années

1980. Ce sont les romans La vengeance de l'orignal (1980), Le trappeur du Kabi (1981) et

Le soleil se lève au Nord (1991) de Doric Germain qui donnent le coup d'envoi au genre du roman d'aventure pour la jeunesse. Ces romans, qui se déroulent dans le Nord de l'Ontario, sont enseignés partout dans la province et il s'agit probablement des œuvres francoontariennes pour la jeunesse les plus étudiées par les universitaires jusqu'à maintenant ${ }^{6}$. Pour Françoise Lepage, « Doric Germain est sans contredit le romancier [pour la jeunesse] le plus “ontarien” dans son inspiration et le plus original de tous. » (2000, p. 431). Ses trois romans

\footnotetext{
${ }^{5}$ L'article « Où en est la littérature franco-ontarienne pour la jeunesse? » publié en 2005 dans la revue Liaison par Françoise Lepage est le dernier article que la chercheuse ait publié sur le sujet avant son décès en 2010. Ainsi, il ne lui aura pas été possible de revenir sur les données présentées qui dressaient effectivement un portrait précaire de la littérature franco-ontarienne pour la jeunesse. Actuellement, comme nous l'indiquent les chiffres présentés plus hauts, la production de la littérature pour la jeunesse franco-ontarienne semble se maintenir, et ce, malgré la diminution des activités des éditions du Vermillon. L'activité dans le secteur littéraire pour la jeunesse se concentrent désormais dans deux maisons d'éditions, soit l'Interligne et David.

${ }^{6}$ On peut notamment songer aux travaux Lucie Hotte (2003, 2012), de Camylle Gauthier-Trépanier (2017) et d'Isabelle Kirouac-Massicotte (2018).
} 
CAMYLLE GAUTHIER-TRÉPANIER, « Nouvelles aventures franco-ontariennes : quelle relève pour la littérature pour la jeunesse en Ontario français? »

pour la jeunesse s'intéressent effectivement à la nature, à la chasse et aux relations avec les communautés autochtones, des thématiques qui se rapprochent de la réalité de la population du nord de l'Ontario. Germain explique être venu à l'écriture pour offrir à ses classes du secondaire, majoritairement composées de garçons, des textes « qui seraient plus proche[s] de leurs préoccupations » (Hotte et Roy, 2012, p. 168) afin de les inciter à lire davantage. Les textes pour la jeunesse de Germain décrivent une nature indomptable, voire dangereuse, mais également un univers manichéen dans lequel le mal ne peut pas triompher : il faut être prêt à apprendre ou mourir comme l'apprendront à leurs dépens les protagonistes de La vengeance de l'orignal et certains de ceux du Trappeur $d u$ Kabi. Les trois récits mentionnés précédemment adoptent une forme assez classique de roman d'apprentissage. Mis bout à bout ces trois textes pourraient même former un cycle d'apprentissage. Ainsi, dans La vengeance de l'orignal, l'apprentissage est un échec et le refus d'apprendre des personnages se solde par leur mort. Dans Le trappeur du Kabi, l'un des personnages est épargné puisqu'il fait preuve de courage et reconnaît également la valeur de son adversaire, qu'il affronte à armes égales. Le soleil se lève au nord n'est le théâtre d'aucun décès. Le protagoniste se montre assez bien disposé à apprendre à vivre avec sa nouvelle communauté puisqu'il n'a nulle part d'autre où aller. En ce qui a trait au lecteur, son apprentissage est facilité par le « système $\mathrm{d}[\mathrm{e}]$ personnages $[\ldots]$ fortement polarisé entre les "méchants" et les "bons" » (Hotte et Roy, 2012, p. 27) mis en place dans l'œuvre de Germain. Toutefois, malgré cette division claire entre le bien et le mal, la justice qui est faite dans ces romans ne découle par des lois ou des institutions qui représente habituellement le bien commun. En effet, elle relève plus souvent de la nature, qui dans ces romans acquiert le statut personnage (Hotte et Roy, 2003, p. 266). Plusieurs protagonistes de Germain outrepassent effectivement les lois humaines pour se 
heurter à l'intransigeance de la nature. C'est le cas dans La vengeance de l'orignal où la cupidité des protagonistes n'est pas freinée par leur comparution devant le tribunal pour s'être adonnés à des pratiques illégales de chasse à l'orignal. Ce n'est qu'après le décès des trois personnages, gelés et noyés, que toute cette aventure est clairement désignée comme une vengeance de la nature puisque celle-ci, « à sa manière, célébrait par cette aurore boréale, la mort du dernier des trois braconniers. » (Germain, 1995 [1980], p. 114)

Par leur peinture plutôt fidèle du Territoire du Nord de l'Ontario, les romans de Germain semblent s'adresser à un lecteur qui connaît ce territoire. Les récits prennent place dans des lieux existants dont l'auteur emploie les véritables noms «pour qu'ils soient facilement repérables. » (Hotte et Roy, 2012, p. 175). Dans La vengeance de l'orignal, par exemple, on évoque la Kabinakagami (Germain, 1995, p. 18) et le Pitukupi (Germain, 1995, p. 18) sans préciser qu'il s'agit d'une rivière et d'un lac. Dans Le trappeur du Kabi, c'est le lac Kabinakagami, souvent désigné comme le Kabi, qui sert de repère spatial. Le lecteur qui ne connaît pas la région devra attendre la page 43 pour comprendre que le lac Kabinakagami est «situé à 130 kilomètres au sud de Hearst [...] [et qu']il se trouve presque au centre du triangle formé par White River, Wawa et Hearst »(Germain, 1993 [1981], p. 43). Le soleil se lève au nord prend entièrement place dans la réserve de Constance Lake, peu d'autres lieux sont donc mentionnés. Au cours du trajet qui le mène à la réserve, le protagoniste croise «quelques agglomérations plus importantes telles Cochrane et Kapuskasing » (Germain, 1991, p. 8) qui ne l'impressionnent guère. Il retient plutôt « les consonances barbares des noms d'Opasatika, Temagami [et] Matachewan »(Germain, 1991, p. 8. Il s'agit des seules mentions de ces villages qu'il appartient au lecteur de connaître ou de situer. Toutefois, il ne s'agit pas seulement de se repérer sur le territoire. Les romans dont il est question ici intègrent 
CAMYLLE GAUTHIER-TRÉPANIER, « Nouvelles aventures franco-ontariennes : quelle relève pour la littérature pour la jeunesse en Ontario français? »

également une composante plus ouvertement didactique qui permet au lecteur de mieux connaître le territoire mis en scène dans le texte. Ainsi, « [c] $]$ haque description est l'occasion de présenter avec maints détails les spécificités géographiques de la région et d'en faire valoir la topographie, la flore et la faune. » (Hotte et Roy, 2012, p. 23)

Ce n'est donc pas dans la découverte du territoire, mais plutôt dans les dangers que dissimule celui-ci que l'on retrouve une forme d'exotisme ou de dépaysement. Toutefois, un lecteur qui ne connaît pas le nord de l’Ontario expérimentera effectivement un dépaysement géographique lors de sa lecture. La question du dépaysement ne peut cependant pas relever du seul territoire, ainsi, elle

ne se résume pas aux effets de couleur locale et à une volonté de satisfaire le goût du lecteur pour l'extraordinaire, [elle] inscrit [plutôt] le récit dans un écart systématique avec le quotidien : non seulement le décor est inaccoutumé, mais les personnages sont excentriques ou exceptionnels, les événements qui se produisent pour le moins inhabituels (Letourneux, 2010, p. 20)

Cette sortie du quotidien qu'évoque Letourneux est présente dans les trois romans de Germain : on découvre un squelette et un pot de pépites d'or dans La vengeance de l'orignal, l'un des personnages est assassiné dans Le trappeur du Kabi et le protagoniste du Soleil se lève au Nord se voit envoyé chez un oncle inconnu à des centaines de kilomètres de chez lui. De plus, en peignant une nature active, voire dangereuse, Germain présente à ses lecteurs un visage insoupçonné d'un monde qui, autrement, leur serait familier. C'est justement ce dynamisme que l'auteur cherche à transmettre à ses lecteurs en leur « laiss[ant] [...] l'idée que, finalement, on n'est pas si mal lotis dans le Nord, même si souvent on est porté à penser qu'on est loin de tout, et que, bon, il n'y a rien ici, alors que pour [lui], au contraire, on est près de ce qui est important [...], la nature. » (Hotte et Roy, 2012, p. 170) 
Sur le plan de la forme, les trois romans de Germain optent pour une narration à la troisième personne du singulier qui est assez commune à la forme du roman d'aventure, lequel délaisse souvent l'intériorité des personnages au profit de l'action. Pour Lucie Hotte et Véronique Roy, cela permet de se distancier des personnages dont la narration n'endosse pas les valeurs (2012, p. 49) comme c'est le cas de ceux de La vengeance de l'orignal et du Trappeur du Kabi. De plus, selon ces chercheuses comme les textes sont destinés à un jeune public, « la narration à la troisième personne permet de formuler de façon plus claire la morale de l'histoire. » (Hotte et Roy, 2012, p. 50). C'est donc davantage par ses thématiques et le décor qu'il choisit pour ses romans, que par ses choix stylistiques que Doric Germain crée des romans susceptibles d'intéresser les jeunes franco-ontariens, particulièrement ceux du nord de la province. Ce faisant, il amène l'aventure à son lecteur plutôt que l'inverse, refusant donc l'idée longtemps prônée par plusieurs chercheurs d'un dépaysement géographique nécessaire au roman d'aventure.

Quoique moins emblématiques de l'Ontario français que les romans de Doric Germain, les textes pour la jeunesse de Jean-Louis Grosmaire constituent également une incursion intéressante du côté du roman d'aventure puisqu'ils se déroulent souvent à l'étranger. Né au Sénégal, Grosmaire vit au Canada, dans la région de l'Outaouais, depuis de nombreuses années et a publié l'entièreté, ou presque, de son œuvre aux Éditions du Vermillon sises à Ottawa. Lucie Hotte et Johanne Melançon ont déjà évoqué la porosité problématique de la frontière outaouaise (Hotte et Melançon, 2010, p. 9) quand vient le temps d'identifier un auteur comme franco-ontarien. Jean-Louis Grosmaire est notamment membre de l'Association des auteures et auteurs de l'Ontario français (AAOF), a fait partie de jurys 
CAMYLLE GAUTHIER-TRÉPANIER, « Nouvelles aventures franco-ontariennes : quelle relève pour la littérature pour la jeunesse en Ontario français? »

littéraires en Ontario et ses romans se sont mérité plusieurs distinctions en Outaouais comme en Ontario ${ }^{7}$.

Les aventures de Louis Travelle, mieux connu comme le Loup, un adolescent français épris d'aventure, se déclinent en cinq tomes. Les deux premiers le mènent au Canada (ParisQuébec, 1992 et Le Loup au Québec, 1997) et les suivants au Vietnam (Paris-Hanoi, 1998), aux États-Unis (Paris-New York, 2002) et au Sénégal (Paris-Saint-Louis du Sénégal, 2005). Les aventures du Loup sont narrées à la première personne du singulier grâce à un calepin dans lequel le personnage note toutes ses aventures. Pour Françoise Lepage il s'agit d'un choix narratif qui contribue à rendre le personnage sympathique aux yeux du lecteur et qui témoigne également de l'influence « des romans réalistes pour adolescents » sur le roman d'aventure (2000, p. 433.) Bien que la narration homodiégétique instaure une forme de connivence entre le lecteur et le personnage, ce dernier se dévoile très peu. On connaît du Loup sa passion pour la culture et la mode américaine ainsi que son goût pour le voyage, deux éléments accentués dans le premier tome de la série, mais cela ne va pas plus loin. L'action occupe définitivement l'avant-plan de cette série dans laquelle les péripéties s'enchaînent très rapidement. Compte tenu du contexte urbain de ces romans, les dangers auxquels doit faire face le Loup se présentent généralement sous la forme de personnages malveillants, comme des trafiquants de drogue. L'univers des romans de Grosmaire s'avère donc, lui aussi, plutôt manichéen, particulièrement en ce qui a trait aux personnages. Toutefois, on observe dans les romans de Germain le souci constant d'une norme morale,

\footnotetext{
${ }^{7}$ Jean-Louis Grosmaire se mérite à deux reprises le prix littéraire Le Droit, en 1993 pour Paris-Québec et en 1999 pour Paris-Hanoi. Son roman Paris-New York sera finaliste pour le même prix en 2003, ainsi qu'au prix Christine-Dumitriu-Van- Saanen (Prix du Salon du livre de Toronto) en 2002. En 2007, le dernier tome de la série, Paris-Saint-Louis du Sénégal, est finaliste au Prix des lecteurs Radio-Canada jeunesse.
} 
incarnée par le respect de la nature, qui doit impérativement être observée par tous sous peine de graves conséquences alors que les romans de Grosmaire reposent, quant à eux, sur la bonne foi des individus et des institutions qui les régissent. Ces dernières, chargées de faire respecter l'ordre moral, sont représentées comme bien plus efficaces dans les romans de Grosmaires que dans ceux de Germain. Règle générale, le personnage ne fait appel que tardivement à la police, mais celle-ci se montre toujours en mesure de l'aider et, surtout, de mettre sous les verrous ceux qui le méritent.

Contrairement aux personnages de Doric Germain chez qui l'aventure survient, toujours inattendue, le Loup provoque ses propres aventures. Ainsi, dans Paris-Québec, il manigance pour partir visiter l'Amérique qu'il rêve de voir depuis toujours et réussit à s'embarquer, sous une fausse identité et à l'insu de sa famille, sur un vol vers Montréal. Dans Paris-Hanoi, il se lance sur la piste d'un mystérieux message trouvé dans un briquet acheté au marché. Le dépaysement géographique, on le comprendra, occupe ici, par l'entremise du voyage, une place de choix. Ainsi, le Loup pose un regard à la fois émerveillé et avide de découvertes sur les pays qu'il visite. À Québec, il se pâme devant les paysages dont il a tant rêvé :

[j]e suis aimanté par la vue. J'arrive à la rencontre du géant de mes rêves. Le fleuve argenté glisse devant moi. Sur l'autre rive, une ville brille au soleil. À gauche, le fleuve se divise en deux et entoure l'île d'Orléans. La chaleur humide estompe les contours. Je pleure. Le vent vient de l'île, portant des bouffées de marées, de pâturage. Des oiseaux blancs crient dans le ciel. (Grosmaire, 1992, p. 159)

La beauté de la nature est également dépeinte par les descriptions de Germain, mais davantage au bénéfice du lecteur que des personnages qui n'y sont que peu sensibles. Chez Grosmaire, le Loup est tour à tour contemplatif et émotif devant les paysages et les cultures qu'il découvre. Il pleure tout naturellement devant le tableau de l'île d'Orléans qui s'offre à 
CAMYLLE GAUTHIER-TRÉPANIER, « Nouvelles aventures franco-ontariennes : quelle relève pour la littérature pour la jeunesse en Ontario français? »

lui, tout comme lorsqu'il entend Diane Dufresne chanter « un souvenir heureux» (Grosmaire, 1992, p. 136) à la radio, au grand désarroi de la camionneuse qui l'accompagne. Lorsqu'il visite le Vietnam avec sa famille dans Paris-Hanoi il est captivé par la ville d'Ho Chi Minh, déclarant que

[1] es touristes devraient se lever tôt le matin, le réveil d'une ville est un spectacle fascinant. Des femmes aux chapeaux coniques, aux pantalons noirs, côtoient des hommes en pantalons bleus et chemises blanches. Ça sent la sauce soja, les légumes bouillis, "avec beaucoup de plantes aromatiques, la coriandre, l'aneth, la citronnelle, le basilic, la menthe, et les épices aux cinq parfums" (Grosmaire, 1998, p. 30).

Les romans de Grosmaire évitent de façon adroite les stéréotypes culturels souvent représentés tant dans le roman d'aventure que dans le roman pour la jeunesse, notamment grâce à des descriptions faisant appel aux sens du lecteur comme c'est le cas ici. Le roman d'aventure se concentre sur l'action et sa fonction première n'est pas de faire découvrir de nouvelles réalités ou culture à son lecteur, mais plutôt de le tenir en haleine. Quant au roman pour la jeunesse, l'usage de stéréotypes est plus souvent lié à la capacité de compréhension du jeune public telle que conçue par les auteurs et éditeurs d'ouvrages pour la jeunesse. En effet, les stéréotypes, s'ils ont le défaut de simplifier certains concepts à l'excès, constituent néanmoins des modèles préexistants qui peuvent faciliter la compréhension du lecteur (Amossy et Herschberg Pierrot, 2005, p. 28). Ainsi, lorsque le Loup débarque à Mirabel dans Paris-Québec, il n'a aucun mal à se faire comprendre par les gens qu'il rencontre. Il n'éprouve pas non plus de difficultés à saisir ce qu'on lui raconte, et ce malgré l'intégration de quelques expressions propre aux francophones du Canada comme «en maudit», « démancher» (Grosmaire, 1992, p. 88) et «Ti-tannant», (Grosmaire, 1992, p. 90). Au contraire, les quelques blagues à ce sujet sont faites aux dépens de l'accent pointu du jeune français. Outre les descriptions des différentes destinations du personnage, une importance 
particulière est accordée aux noms des lieux, dans une volonté peut-être de situer le lecteur ou alors de montrer que le personnage doit faire un effort pour s'orienter et ne pas s'égarer dans des lieux qu'il ne connaît pas. Ainsi, on mentionne divers noms de rues ou encore de petits villages au fil des aventures du Loup.

Les romans de Doric Germain et de Jean-Louis Grosmaire, s'ils sont bien des romans d'aventure, empruntent néanmoins des voies fort différentes. On observe cependant un certain nombre d'éléments récurrents chez ces deux auteurs, notamment la question des lieux et de la conscience de l'environnement. Chez Doric Germain en particulier le désir de situer le lecteur est omniprésent. Il faut dire que cela permet aussi de faire entrer dans le domaine de la fiction des lieux qui, pour les lecteurs du nord de l'Ontario, évoquent plutôt quelque chose de commun. Grosmaire cherche également à situer son lecteur en lui fournissant certains détails comme des noms de rue, des adresses, les noms de très petits villages, mais il ne s'agit pas toujours d'endroits reconnaissables ou réels comme chez Germain. Ces informations que doit retenir ou découvrir le protagoniste ajoutent à la vraisemblance du roman et donnent l'impression de pouvoir suivre le Loup à la trace. Les deux auteurs rivalisent d'ingéniosité pour mettre en scène les aventures de leurs personnages dans le contexte le plus réaliste possible sans remettre en question l'aspect extraordinaire de ces événements, pensons par exemple à la découverte de l'or dans La vengeance de l'orignal, au GI amnésique dans Paris-Hanoi ou à la vendetta du métis dans Le trappeur du Kabi. Ainsi, les rouages des plans du Loup sont explicités au bénéfice du lecteur et chaque obstacle est contourné d'une façon logique. Chez Doric Germain, on prend le temps d'expliquer le fonctionnement d'outils ou encore les différents défis que pose la forêt pour les projets de personnages. Enfin, les deux auteurs laissent une place importante à la description de 
CAMYLLE GAUTHIER-TRÉPANIER, « Nouvelles aventures franco-ontariennes : quelle relève pour la littérature pour la jeunesse en Ontario français? »

l'environnement. Chez Germain, ces descriptions imposent le respect puisque la forêt doit être à la fois crainte et respectée. Chez Grosmaire, elles emplissent le Loup d'émotion et le touchent profondément.

\section{Le roman d'aventure depuis 2010}

Doric Germain et Jean-Louis Grosmaire semblent aujourd'hui avoir tous les deux délaissé l'avenue du roman pour adolescents. C'est donc par l'entremise de deux auteurs dont les textes ont été publiés après 2010, soit Pierre-Luc Bélanger et Daniel Marchildon, que nous tenterons d'appréhender le roman d'aventure tel qu'il s'écrit aujourd'hui et d'en cerner l'évolution par rapport aux ouvrages analysés précédemment. Bien que Daniel Marchildon publie activement depuis le début des années 1990, Otages de la nature constitue sa première incursion dans le genre du roman d'aventure tel que nous le concevons ici. Nous considérons qu'il pourrait s'agir d'un texte de la relève puisque l'auteur appréhende les modalités d'un genre littéraire différent et doit nécessairement, tenir compte du contexte actuel. De son côté Pierre-Luc Bélanger entame sa carrière d'auteur avec la publication, en 2013, de 24 heures de liberté qui sera rapidement suivi de trois autres ouvrages Ski, Blanche et avalanche ${ }^{8}$ (2015), Disparue chez les Mayas (2017) et L'Odyssée des neiges (2018). La récente venue à l'écriture de celui-ci, ainsi que le nombre de ses publications laisse à penser qu'il se taille déjà une bonne place sur la scène de la littérature pour la jeunesse franco-ontarienne. Dans 24 heures de liberté (2013), son premier roman, un adolescent et sa jeune sœur, placés en famille d'accueil après qu'un drame se soit abattu sur leur famille, s'échappent pour retrouver leur père, emprisonné. Le roman suit le périple qu'entame Sébastien pour retrouver sa sœur,

\footnotetext{
${ }^{8}$ Ski, Blanche et avalanche a remporté le prix Le Droit jeunesse 2016, le prix de la Toronto French School 2016 et le prix Trillium du livre d'enfant 2017.
} 
Annie, qu'il soupçonne d'être maltraitée dans sa famille d'accueil. Le frère et la sœur entament ensuite un périple qui les mènera d'Orléans, en banlieue d'Ottawa, jusqu'au pénitencier de Kingston. La majeure partie de l'ouvrage se déroule en milieu urbain, ce qui confère à l'aventure de Sébastien et d'Annie beaucoup de réalisme, malgré les défis que cela représente, notamment lorsque les protagonistes doivent se déplacer à travers la ville sans argent. Ce roman peint un portrait très réaliste pour les lecteurs franco-ontariens de l'Est de la province. On note une volonté omniprésente de situer le lecteur dans l'espace. La narration évoque ainsi de nombreux repères facilement identifiables de la région d'Ottawa, c'est le cas des stations d'autobus comme «l'arrêt campus » (Bélanger, 2013, p. 70) et de bâtiments reconnaissables comme « l'hôpital Monfort » (Bélanger, 2013, p. 56) et le «Centre national de recherche du Canada [...] à l'angle de la rue Blair » (Bélanger, 2013, p. 59). Ces repères sont évoqués de façon familière et semblent s'adresser à un lecteur qui connaît bien la ville d'Ottawa et ses environs. On le voit bien lorsque les personnages décident de faire de l'autostop pour franchir la distance entre Ottawa et Kingston :

Annie et Sébastien contemplaient l'autoroute devant eux. À cette heure-là, il y avait peu de voitures en direction de Kanata, et même en sens contraire. La circulation était raisonnable. Les jeunes devraient traverser la 417 à cette fourche en « $\mathrm{y} »$ pour suivre l'autoroute 416 jusqu'à ce qu'elle rejoigne la 401.

Les numéros des autoroutes permettent de reconstituer facilement le trajet que devront faire les personnages pour atteindre le pénitencier de Kingston. Toutefois, le lecteur qui connaît la région sera plus en mesure de saisir le danger de cette manœuvre puisque l'autoroute 417 compte à cette hauteur quatre voies dans un sens comme dans l'autre. Cette insistance à situer le lecteur de façon aussi précise semble témoigner d'un désir de s'adresser à un public précis, soit les jeunes ottaviens, ou alors d'une volonté de se distancier de l'étiquette «nordique » 
CAMYLLE GAUTHIER-TRÉPANIER, « Nouvelles aventures franco-ontariennes : quelle relève pour la littérature pour la jeunesse en Ontario français? »

souvent apposée au roman pour la jeunesse franco-ontarien qui est fréquemment réduit aux œuvres de Doric Germain et d'Hélène Brodeur. Le décor urbain et actuel du roman contribue effectivement à s'éloigner des univers de ces deux auteurs.

En termes de style toutefois, Bélanger se rapproche un peu de Doric Germain. Raymond Bertin évoque dans son compte rendu de 24 heures de liberté « une écriture sans fioriture [...] [qui] parvient à bien installer les situations. » (Bertin, 2014, p. 45) Toutefois, la précision et l'efficacité de l'écriture de Bélanger ne laissent que peu de place à l'imagination du lecteur (Bertin, 2014, p. 45). Il n'y a pas de mystère qui plane dans 24 heures de liberté, Annie et Sébastien savent qu'ils disposent de peu de temps pour rejoindre leur père à Kingston. Ils savent également qui sont leurs poursuivants, soit la police et les services à l'enfance. Ainsi, alors que le lecteur craint ce qui pourrait arriver aux protagonistes de $L a$ vengeance de l'orignal et du Trappeur du Kabi, l'écriture de Bélanger met plutôt en place un sentiment d'urgence qui cadre bien avec le récit. Cette impression d'urgence est accentuée par l'enchaînement extrêmement rapide des événements qui crée ainsi, selon les critiques, « un rythme effréné » (s.a., 2014), voire essoufflant (Bertin, 2014, p. 45). La structure de l'ouvrage contribue également à instaurer ce rythme plus que soutenu avec ses 24 courts chapitres composés de quelques pages seulement. La narration à la troisième personne permet ici de porter un regard d'ensemble sur les remous que causent la fuite des deux jeunes, notamment grâce à la focalisation de cette narration sur différents personnages comme JeanCharles Laframboise, l'employé de la Société d'aide à l'enfance responsable du dossier d'Annie et de Sébastien, et la constable Larocque de la police municipale d'Ottawa. Toutefois, on note, malgré la narration à la troisième personne du singulier, l'instauration d'une forme de familiarité entre le narrateur et le personnage principal, Sébastien qu'il 
désigne par son surnom, «Sébas ». En ce qui a trait aux personnages, on note tout de suite la présence de plusieurs femmes aux rôles significatifs, dont la petite sœur et la mère, qui, après être sortie du coma, subvient aux besoins de sa famille seule et parvient même à leur acheter une maison (Bélanger, 2013, p. 128).

De son côté, Otages de la nature de Daniel Marchildon propose une version actualisée de la forme du roman d'aventure en y intégrant des enjeux écologiques ainsi qu'une diversité de points de vue. Le roman s'ouvre sur le personnage d'Alex et de sa mère, Fleur, chanteuse en panne d'inspiration, qui doivent s'arrêter à la pourvoirie de l'écho vert en attendant de faire réparer leur voiture. Le propriétaire, David, leur apprend que l'écosystème de la région, de même que l'emplacement des dunes sacrées de la communauté Anishnabé, est menacé par une entreprise de foresterie. Alex et Fleur, convaincus par la nature dont ils font l'expérience, se rangeront aux côtés de David dans cette lutte écologique. L'ouvrage prend ses distances avec les structures plus classiques du roman d'aventure en adoptant, d'une part, une narration à la première personne du singulier et, d'autre part, deux narrateurs aux points de vue complémentaires : Alex et Danika, une jeune militante Anishnabé. L’aventure ellemême se trouve là où les personnages ne l'attendent pas. Ils sont, bien sûr, dépaysés par tout ce qu'ils découvrent, notamment sur le conflit opposant les militants écologistes à l'entreprise forestière, mais ne marchent pas vers l'aventure, à la différence du personnage de Bélanger. C'est plutôt cette dernière qui vient à eux. Ainsi, «Marchildon entremêle avec succès aventure, écologie, politique, relations familiales complexes, amour et amitié, ainsi que culture autochtone et franco-ontarienne. Le récit nous tient en haleine, car chaque chapitre nous laisse en suspens. » (Brisson, 2018, p. 63) Le titre de chaque chapitre reprend d'ailleurs 
CAMYLLE GAUTHIER-TRÉPANIER, « Nouvelles aventures franco-ontariennes : quelle relève pour la littérature pour la jeunesse en Ontario français? »

la dernière phrase dudit chapitre, laissant le lecteur avide de savoir ce qui se cache derrière ces phrases énigmatiques.

Marchildon campe son roman « dans la forêt d'un village franco-ontarien fictif dans le Nord de l'Ontario : Rivière Ahmic. » (Radio-Canada.ca, 2018) Il ne dresse pas de portrait précis de cette localité. Toutefois, plusieurs autres éléments permettent de rappeler, que l'on se trouve en Ontario comme des agents de la Police provinciale de l'Ontario (Marchildon, 2018, p. 13), «les immenses lettres jaunes, OPP ${ }^{9}$, [qui] recouvrent le ventre [de l'hélicoptère] » (Marchildon, 2018, p. 73), les mentions de la ville de Toronto (Marchildon, 2018, p. 48) et du journal Toronto Star (Marchildon, 2018, p. 79). C'est la nature, jusque-là inconnue, qui va réellement faire entre Fleur et son fils dans un tout autre univers et les dépayser. À la vue des dunes, le jeune narrateur déclare que « [1]e spectacle [1]e laisse bouche bée. Devant [Fleur] se dresse une haute colline de sable brun. Contrairement à une oasis, un trou d'eau dans le milieu d'un désert, [ils] so[nt] devant un monticule de désert au milieu d'une forêt luxuriante. (Marchildon, 2018, p. 42) Ici, l'émerveillement va de pair avec la spiritualité remarque le narrateur : «Le lieu est spécial, unique, cela ne fait aucun doute. J'étudie les grains de sable soulevés par la brise soudainement plus forte. Ici, même l'air transforme le son du vent pour créer une musique indescriptible. » (Marchildon, 2018, p. 42)

Les deux camps présentés dans le roman défendent des positions qui demeurent actuelles à savoir celles du progrès ou du développement économique et celle de la conscience environnementale, revisitant ainsi paradigme nature/civilisation évoqué par Hotte et Roy (2012, p. 42-44) à propos des romans de Doric Germain. Bien que la nature revête

\footnotetext{
${ }^{9}$ OPP est l'abréviation utilisée pour Ontario Provincial Police.
} 
dans ce roman une importance capitale pour les défenseurs des dunes, auxquels se rallient Alex et Fleur, elle n'acquiert pas, comme chez Germain, un statut de personnage. Par conséquent, l'univers du roman est soumis aux lois humaines et les personnages doivent subir les conséquences de leurs actes. Ainsi, David, ira en prison pour avoir orchestré une prise d'otages avec l'aide de Fleur et d'Alex. Cette question des gestes à poser pour défendre l'environnement est d'ailleurs centrale au projet de l'auteur qui désire pousser ses lecteurs à réfléchir aux moyens employés par les personnages pour défendre leurs convictions ainsi qu'aux actions qu'eux-mêmes peuvent poser.

\section{Une relève en chantier}

En somme, les ouvrages de Pierre-Luc Bélanger et de Daniel Marchildon permettent de mettre en lumière un certain nombre d'éléments qui n'apparaissent pas, ou très peu, chez leurs prédécesseurs et qui pourraient constituer les éléments d'une esthétique de la relève dans le roman d'aventure franco-ontarien pour la jeunesse. Il s'agit de l'inscription du territoire ontarien urbain dans le texte, de la mise en scène d'adolescents à la frontière de l'âge adulte, de la présence de personnages féminins qui étaient généralement absents chez Doric Germain et Jean-Louis Grosmaire, ainsi que de certaines structures narratives empruntées au roman socioréaliste, lesquelles sont ici illustrées davantage dans Otages de la nature, mais se présentent également dans les autres romans de Bélanger ${ }^{10}$. Toutefois, la proposition principale de ces deux auteurs semble viser à réinscrire le roman d'aventure dans la société telle que nous la connaissons. Dans les romans de Doric Germain, la nature supplante les autorités humaines. C'est d'ailleurs la nature, et non les lois humaines bien que

\footnotetext{
${ }^{10}$ Ski, Blanche et avalanche (2015), Disparue chez les Mayas (2017) et L'Odyssée des neiges (2018)
} 
CAMYLLE GAUTHIER-TRÉPANIER, « Nouvelles aventures franco-ontariennes : quelle relève pour la littérature pour la jeunesse en Ontario français? »

toutes deux tendent parfois vers un même objectif, que doivent respecter les personnages s'ils veulent s'en sortir indemne. Chez Grosmaire les lois semblent très flexibles et le personnage du Loup n'a jamais vraiment à s'y confronter puisqu'il est toujours du bon côté des choses. Toutefois, il est surprenant que plusieurs de ses actes demeurent impunis, particulièrement dans le premier tome de la série où il fugue, et emprunte une identité pour voyager. À titre d'exemple, on notera que, pour avoir libéré sa sœur d'un foyer d'accueil où elle était maltraitée et avoir fui la police, le protagoniste de 24 heures de liberté devra effectuer des heures de services communautaires puisqu'il s'agit d'infractions à la loi. Otages de la nature reproduit un scénario similaire puisqu'à la suite de la fausse prise d'otages, le personnage de David est emprisonné pour une durée d'un an. Bien qu'il n'ait pas été accusé de prise d'otages, «il a été poursuivi pour méfait public, menace contre des agents de police et utilisation négligente d'une arme à feu. Toutes les lettres de sympathie que l'inculpé a reçues et même la presse favorable n'ont pas pu faire fléchir le juge qui [...] [a] tenu à appliquer la loi » (Marchildon, 2018, p. 106).

La question de la représentation de l'Ontario et, dans une certaine mesure, d'éléments liés à l'identité franco-ontarienne est également très intéressante puisque cela permet de distinguer ces ouvrages par rapport à la production québécoise sans tomber dans les stéréotypes. L'Ontario, ce n'est pas qu'une forêt et des lacs comme le présentent les romans de Doric Germain, c'est aussi tout un monde urbain et des communautés différentes qui cohabitent. Chacun à leur façon, Pierre-Luc Bélanger et Daniel Marchildon peignent un portrait de l'Ontario qui, sans nécessairement s'opposer à celui de Germain, s'en éloigne et intègre un certain nombre d'éléments qui permettent de le complexifier. Ainsi, les référents ne sont plus seulement géographiques. Ils ont désormais une valeur sociale ou culturelle. 
C'est le cas, par exemple, lorsque sont évoqués les journaux Le Droit et le Ottawa Citizen dans le roman de Bélanger ou encore la Police provinciale de l'Ontario et le Toronto Star chez Marchildon. Ces éléments, évocateurs pour des lecteurs franco-ontariens, ne permettent pas nécessairement de situer l'action à proprement parler, mais ils constituent des rappels subtils de l'Ontario dans des romans dont l'objectif est de raconter des aventures plutôt que de raconter l'Ontario français.

Nous évoquions plus tôt que les textes de la relève ne peuvent finalement se définir que par rapport à ceux qui les ont précédés en s'en inspirant pour les dépasser ou les surpasser de quelque façon que ce soit. La production littéraire des années constituerait pour Marchildon et Bélanger un tremplin vers une conception actuelle, une relève, du roman d'aventure franco-ontarien pour la jeunesse. Les textes de semblent effectivement entretenir des liens avec les romans d'aventure des années 1990, même si ceux-ci s'inscrivent parfois dans une dynamique d'opposition. Ainsi, le texte de Bélanger s'oppose à l'idée de représenter la nature de l'Ontario, omniprésente chez Doric Germain, pour plutôt chercher à décrire un décor urbain connu du lecteur. Cela confère une originalité certaine au texte puisque peu d'ouvrages pour la jeunesse représentent Ottawa et encore moins le font avec une telle précision. Marchildon, lui, choisit de conserver un décor forestier, mais développe plutôt une réflexion par rapport au territoire et à la flore au moyen de considérations écologiques et culturelles. Des éléments liés à l'écologie et à la protection de la nature avaient été relevés par Lucie Hotte et Véronique Roy dans les textes de Doric Germain, mais ceux-ci ne faisaient pas partie du projet de l'auteur (Hotte et Roy, 2012, p. 170-171). L'intégrations des personnages féminins dans les deux romans constitue peut-être l'un des aspects les plus originaux de ces textes par rapport à ceux des années 1990 dans lesquels elles étaient 
CAMYLLE GAUTHIER-TRÉPANIER, « Nouvelles aventures franco-ontariennes : quelle relève pour la littérature pour la jeunesse en Ontario français? »

quasiment absentes ${ }^{11}$. On le voit particulièrement bien dans Otages de la nature où le personnage de Danika est chargée d'une partie de la narration du roman. Ainsi, la relève du roman franco-ontarien pour la jeunesse existe bel et bien, mais elle n'obtient peut-être pas la même visibilité que les romans des années 1990, en partie à cause de la cessation, en 2018, des activités de la revue Liaison qui proposait des comptes rendus d'œuvres pour la jeunesse franco-canadiennes. La littérature pour la jeunesse franco-ontarienne actuelle, ne semble pas non plus avoir suscité l'intérêt chez les chercheurs universitaires. Il est donc difficile de comprendre si cela est dû aux romans actuels ou a un manque d'intérêt pour l'étude de la littérature pour la jeunesse. Les analyses menées précédemment montrent bien que les textes de Pierre-Luc Bélanger et de Daniel Marchildon parviennent à s'inspirer des textes des années 1990 pour construire des univers plus réalistes (Bélanger) et nuancés (Marchildon) qui sont susceptible de parler aux lecteurs d'aujourd'hui. Comme ceux de Doric Germain, le roman de Pierre-Luc Bélanger décrit avec précision un endroit connu de nombreux francoontariens, reprenant cette idée d'inscrire différents espaces de l'Ontario dans la littérature Une idée qui a grandement contribué à l'originalité de Germain. Chez Daniel Marchildon, ce sont les questions d'écologie et de la culture de l'autre qui font l'originalité du texte, plus particulièrement parce que le roman donne véritablement la parole aux groupes concernés, ce que ne faisaient pas les romans de Grosmaire et de Germain. L'originalité des textes est indiscutable et la qualité peut même être attestée par les prix reçus par l'ouvrage de Bélanger,

\footnotetext{
${ }^{11}$ Dans les aventures du Loup (Grosmaire) on observe à quelques reprises le personnage de la mère, mais elle occupe un rôle secondaire. Chez Doric Germain, il n'y a qu'un personnage féminin très secondaire dans Le trappeur du Kabi. Le soleil se lève au Nord met en place deux personnages de femmes, la tante du personnage principal et Mona, de laquelle il tombera amoureux. Toutefois, Lucie Hotte et Véronique Roy indiquent que la fonction principale de Mona est de donner au lecteur à observer la transformation du personnage principal tout au long du roman (Hotte et Roy, 2012, p. 137).
} 
celui de Marchildon étant encore trop récent. La relève existe en littérature pour la jeunesse franco-ontarienne, mais pour qu'elle continue de le faire, il faut en parler, il faut l'étudier et cela doit passer à la fois par les études en littérature et par le domaine de l'éducation. L'existence de plateformes dédiées à l'enseignement de la littérature comme FousdeLire.ca (2014) et LireenOntario.ca (2018), de même que diverses initiatives du Regroupement des éditeurs franco-canadiens et du Centre franco-ontarien de ressources pédagogiques contribuent grandement à faire connaître la littérature pour la jeunesse franco-ontarienne et à l'intégrer dans le parcours scolaire. Les études littéraires ont également un rôle à jouer dans la légitimation du livre pour la jeunesse franco-ontarien en l'enseignant dans les universités et en l'étudiant. C'est une combinaison de ces deux champs qui permettra à la relève de la littérature pour la jeunesse d'occuper une place d'importance sur la scène littéraire.

Ainsi, on pourra en conclure que le portrait de la littérature franco-ontarienne pour la jeunesse aura tout de même changé un peu depuis les succès de Doric Germain et Jean-Louis Grosmaire. La création de la collection «14/18 » des éditions David a concentré davantage la production littéraire pour la jeunesse dans la région d'Ottawa. Cela pourrait éventuellement rendre cette production plus représentative des franco-ontariens de l'Est de la province, tout comme les romans de Doric Germain puis d'Hélène Brodeur étaient plus emblématiques du nord de la province. Bien que les différentes maisons d'éditions basées à Ottawa publient principalement des auteurs de partout en Ontario et même d'autres provinces canadiennes, on note également la présence de nombreux auteurs de l'Outaouais au sein de celles-ci. Il est par ailleurs difficile d'établir clairement la provenance des auteurs dans cette région et donc de distinguer nettement de grandes tendances ou mouvements qui s'étendraient à l'ensemble du roman franco-ontarien pour la jeunesse, par exemple. Cela renforce la nécessité d'intégrer 
CAMYLLE GAUTHIER-TRÉPANIER, « Nouvelles aventures franco-ontariennes : quelle relève pour la littérature pour la jeunesse en Ontario français? »

des référents destinés à des lecteurs franco-ontariens dans les ouvrages pour la jeunesse et surtout de trouver un équilibre entre l'identité de laquelle se réclament l'ouvrage et son auteur ainsi que le récit lui-même.

\section{Bibliographie}

S. A. (14 avril 2014), « 24 heures de liberté », La Recrue, [En ligne]. [https://larecrue.org/24heures-de-liberté-27d483cbb90d] (Consulté le 3 janvier 2019).

Amossy, Ruth et Anne Herschberg PIERrot (2005), Stéréotypes et clichés : langue, discours, société, Paris, Éditions Armand Colin, coll. «128».

BÉlANGER, Pierre-Luc (2013), 24 heures de liberté, Ottawa, Éd. David, coll. « 14/18», [Epub].

BERTIN, Raymond (2014), « 24 heures de liberté », Lurelu, vol. 37, no. 1, p. 45.

Brisson, Geneviève (2018), «Otages de la nature », Lurelu, vol. 41, no. 2, p. 63.

CENTRE DE RECHERCHE EN CIVILISATION CANADIENNE FRANÇAISE (2003). «L'Imprimerie Beauregard », La présence française en Ontario : 1610, passeport pour 2010, [En ligne]. [https://crccf.uottawa.ca/passeport/II/A2a/IIA2a01-1.html] (4 avril 2019).

GERMAIN, Doric (1991), Le soleil se lève au Nord, Sudbury, Éd. Prise de parole, coll. «Roman ».

GERMAIN, Doric (1993 [1981]), Le trappeur du Kabi, Sudbury, Éd. Prise de parole, coll. « Roman ».

GERMAIN, Doric (1995 [1980]), La vengeance de l'orignal, Sudbury, Éd. Prise de parole, coll. « Roman ».

GrosmaIRE, Jean-Louis (1992), Paris-Québec, Ottawa, Éd. du Vermillon.

Grosmaire, Jean-Louis (1998), Paris-Hanoi, Ottawa, Éd. du Vermillon.

HotTe Lucie et Johanne MELAnÇON (dir.) (2010), Introduction à la littérature francoontarienne, Sudbury, Prise de parole, coll. « Agora ». 
HotTe, Lucie et François OuEllet (dir.) (2016), La littérature franco-ontarienne depuis 1996. Nouveaux enjeux esthétiques, Sudbury, Prise de parole, coll. « Agora ».

Hotte, Lucie et Véronique Roy (2003), « Devenir homme : l'apprentissage de la vie dans les romans pour la jeunesse de Doric Germain », dans Françoise Lepage (dir.), La littérature pour la jeunesse 1970-2000, Montréal, Fides, coll. " Archives des lettres canadiennes », 2003, p. 265-286.

HotTe, Lucie et Véronique Roy (2012), Doric Germain, Ottawa, Éd. David, coll. « Voix didactiques ».

LEPAGE, Françoise (2000), Histoire de la littérature jeunesse : Québec et francophonies du Canada, Orléans, Éd. David.

LEPAGE, Françoise (2005), « Où en est la littérature franco-ontarienne pour la jeunesse? », Liaison, no. 129, p. 68-71.

LetourneuX, Matthieu (2010), Le roman d'aventures : 1870-1930, Limoges, Presses universitaires de Limoges, coll. «Mediatextes».

Marchildon, Daniel (2018), Otages de la nature, Ottawa, Éd. David, coll. «14/18», [Epub].

PARÉ, François (2001 [1992]), Les littératures de l'exiguïté, Ottawa, Le Nordir, coll. «Bibliothèque canadienne française ».

RADIO-CANADA.CA (10 février 2018). «Deux nouveaux livres de l'auteur franco-ontarien Daniel Marchildon», Grands Lacs café, [En ligne]. [https://ici.radiocanada.ca/premiere/emissions/grands-lacs-cafe/segments/entrevue/58801/otages-naturelivre-marchildon] (Consulté le 5 janvier 2019).

\section{Résumé}

S'inspirant des travaux de Françoise Lepage et de ses réflexions sur la relève en littérature pour la jeunesse, cette étude s'intéresse au roman d'aventure franco-ontarien pour la jeunesse et à son évolution depuis les années 1980. Nous cernons d'abord, à l'aide des œuvres de Doric Germain et de Jean-Louis Grosmaire, les particularités du roman d'aventure pour la jeunesse tel qu'il s'est écrit en Ontario français dans les décennies 1980 et 1990. Par la suite, nous analysons un échantillon de la production littéraire plus récente, afin d'observer l'évolution des caractéristiques dégagées précédemment et d'identifier des éléments propres aux romans formant la relève du roman d'aventures franco-ontarien pour la jeunesse aujourd'hui. Plus largement, cet article réfléchit à la littérature franco-ontarienne pour la jeunesse, à sa diffusion et à la place qu'elle occupe dans les espaces universitaires et critiques. 


\begin{abstract}
Inspired by Françoise Lepage's work and her thoughts on emerging children's and youth literature, the present study focuses on the Franco-Ontarian adventure novel for teen readers and its evolution since the 1980s. Using novels by Doric Germain and Jean-Louis Grosmaire, we are, at first, identifying the particularities of the Franco-Ontarian adventure novel for teens as it was written in French Ontario in the 1980s and 1990s. Thereafter, we analyze two novels published after 2010 to observe the evolution of the previously identified characteristics and see if new elements now define the emerging literary production of the Franco-Ontarian adventure novel for teens. More broadly, this article reflects on Franco-Ontarian literature for teens, its diffusion and the place it holds in academic and critical spaces.
\end{abstract}

\title{
Notice bibliographique
}

Camylle Gauthier-Trépanier est doctorante à l'Université d'Ottawa où elle détient une bourse Joseph Armand Bombardier du Conseil de recherche en sciences humaines du Canada. Elle a complété une maîtrise en lettres françaises à l'Université d'Ottawa au cours de laquelle elle a étudié l'évolution de la quête initiatique dans les romans pour la jeunesse franco-canadiens. Ses recherches actuelles portent sur les représentations de la violence dans les romans pour adolescents au Canada francophone et au Québec. 